\title{
CFD-Simulation zur Untersuchung des Strömungsverhaltens in Strukturrohren*
}

\author{
Udo Hellwig, Hartwig Nikolaus
}

\section{Ausgangssituation}

Die CFD (Computational Fluid Dynamics)-Simulation hat mittlerweile in alle technischen Bereiche Einzug gehalten, in denen die Vorhersage strömungstechnischer Ereignisse relevant ist. Nach wie vor ist allerdings eine Validierung von Rechenergebnissen mit Messdaten unerlässlich, um zu vertrauenswürdigen Simulationsergebnissen zu kommen. Dies ist u. a. darauf zurückzufuihren, dass die am Markt verfuigbare CFD-Software darauf ausgerichtet ist, ein möglichst breites Anwendungsspektrum zu bedienen. Die implementierten Berechnungsmodelle müssen im Rahmen der Modellierung durch die Wahl geeigneter Randbedingungen an den zu untersuchenden Anwendungsfall angepasst werden.

\section{Untersuchungsgegenstand}

Im Rahmen eines mehrjährigen anwendungsorientierten Projektes zur Entwicklung von Bauteilen für Abgasanlagen in Fahrzeugen, gefördert durch die Deutsche Bundesstiftung Umwelt (DBU), wurden uiber die Firma Steremat Elektrowärme in Berlin die Strömungs- und Wärmeübertragungseigenschaften von Industrial Power Tubes, kurz IP-Tubes genannt, untersucht. IP-Tubes sind gekennzeichnet durch eine vom Herstellungsprozess vorausbestimmbare Anzahl von Einbeulungen (Nebenformen) bezogen auf den Rohrumfang (Hauptform). Anzahl, Tiefe, axialer und rotatorischer Versatz der Nebenformen können in definierten Grenzen beim Herstellungsprozess variiert werden. Die IP-Tubes sollen wegen ihrer besonderen akustischen Eigenschaften (selektive Dämmwirkung in bestimmten Frequenzbereichen) in Kfz-Abgasanlagen eingesetzt werden. In modernen Abgasanlagen sind zumindest zwei weitere Bereiche von Bedeutung, die eine partielle Substitution von Glattrohren durch IP-Tubes sinnvoll erscheinen lassen. Diese Bereiche sind unmittelbar mit der Beaufschlagung des Katalysators durch den Abgasstrom gekoppelt. Da die Katalysatoren nur in einem engen Temperaturfenster hohe Umsatzraten gewährleisten können, muss bei bestimmten Fahrzeugen die Abgastemperatur nach dem Austritt aus dem Motor auf einer kurzen Strecke möglichst stark heruntergekuihlt werden. An dieser Stelle können die besonderen Wärmeübertragungseigenschaften der IP-Tubes genutzt werden. Hinzu kommt, dass die Anströmung des Katalysators mit über den erzielbaren Umsatzgrad entscheidet. Da oftmals scharfe Umlenkungen der Abgasanlage vor dem Eintritt

\footnotetext{
* gefördert durch die Deutsche Bundesstiftung Umwelt (DBU) als Teilprojekt des Vorhabens ,Entwicklung von strukturierten Kfz-Abgasanlagenelementen mit verbesserten Gebrauchs- und Umweltparametern“
}

in den Katalysator erforderlich sind, um die Abgasanlage in das gesamte Fahrzeugkonzept zu integrieren, entsteht eine ungleichmäßige Geschwindigkeitsverteilung am Eintritt des Katalysators. Durch die IP-Tubes kann ggf. eine Vergleichmäßigung der Geschwindigkeitsverteilung erfolgen.

\section{Aufgabenstellung und Lösungsstrategie}

Im ersten Schritt wurde ein geeigneter Modellansatz zur Berechnung von IP-Tubes erstellt und mit verfügbaren Berechnungsansätzen für Glattrohre validiert. Insbesondere das Rechenverfahren und notwendige Adaptionen des Berechnungsgitters standen bei der Erarbeitung eines geeigneten Lösungsverfahrens im Vordergrund. Durch einen Vergleich der IP-Tubes mit auf dem Markt verfuigbaren Wettbewerbsprodukten wird die Abgrenzung der IP-Tubes ermöglicht. Verglichen wurden die IP-Tubes mit Drallrohren, für die verlässliche Messdaten zur Validierung der Berechnungsergebnisse vorliegen. Berechnungen der für Abgasanlagen typischen Randbedingungen stellen den Bezug zu dem zu untersuchenden Anwendungsfall her. Eine Untersuchung von komplett strukturierten Abgasanlagenelementen wurde verworfen, da nur partiell strukturierte Elemente zur Anwendung kommen werden. Aus diesem Grund wurde unter Berücksichtigung der durch die Umformeinrichtung gegebenen Restriktionen bezüglich herstellbarer Oberflächengeometrien eine Versuchsreihe zur Auswirkung verschiedener Strukturierungsvarianten auf die Zielgrößen Wärmeübertragung und Druckverlust für einzelne Rohrabschnitte untersucht.

\section{Randbedingungen für IP-Tubes-Simulation}

Ein wesentliches Problem bei der mathematischen Beschreibung von turbulenten Strömungen besteht darin, den Übergang von der turbulenten Kernströmung (viskositätsfrei), die durch Turbulenzmodelle hinreichend beschrieben werden kann, zur Wand (viskositätsdominiert) darzustellen. Dieser als Grenzschicht bezeichnete Übergangsbereich wird bei der Berechnung durch sogenannte Wandgesetze überbrüickt, um den Berechnungsaufwand zu minimieren.

\subsection{Wandfunktionen}

Prinzipiell besteht die Möglichkeit, die viskose Unterschicht durch ein hinreichend feines Gitter aufzulösen und die Randbedingungen entsprechend zu wählen (vgl. Abb. 1: Near-Wall Model Approach). Hierzu ist es notwen- 
dig, die viskose Unterschicht zumindest mit einer Schichtdicke von ca. zehn Zellen aufzulösen, da die Gradienten in Wandnähe sehr stark sind und nur durch ein extrem feines Gitter aufgelöst werden können. Mit zunehmender Reynolds-Zahl nimmt die Schichtdicke der viskosen Unterschicht durch Verdrängung ab, wodurch das wandnahe Gitter immer feiner werden muss, was mit einem starken Anstieg des Berechnungsaufwandes einhergeht.

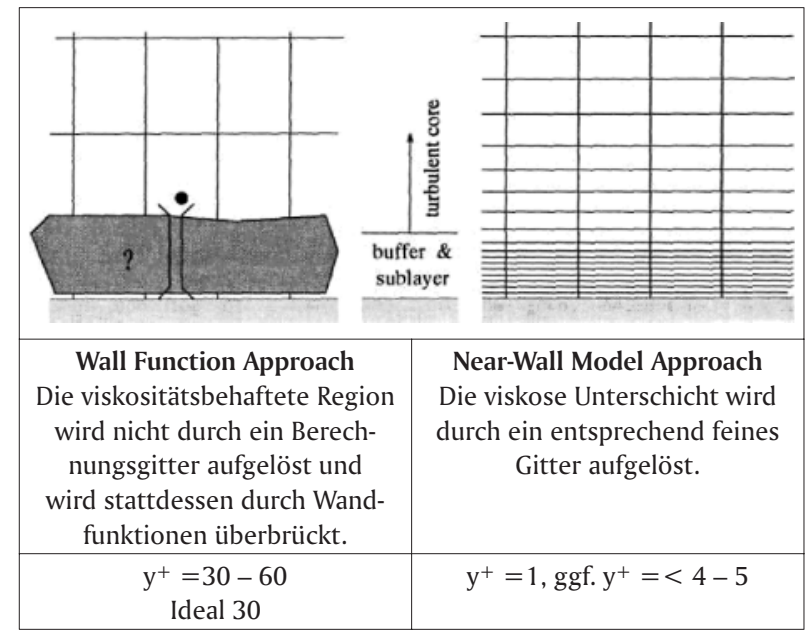

Abb. 1: Vernetzungsstrategien für unterschiedliche Berechnungsansätze

Durch Wandfunktionen kann die Grenzschicht der Strömung in Wandnähe überbrückt werden, da in einer vollturbulenten Strömung die Geschwindigkeit außerhalb der viskosen Unterschicht logarithmisch anwächst.

$\mathrm{u}^{+}=\frac{1}{\mathrm{~K}} \ln \mathrm{y}^{+}+\mathrm{B}$

Gleichung 1

Hier sind $\mathrm{y}^{+}$und $\mathrm{u}^{+}$normierte Größen für den Wandabstand $\partial$ bzw. die tangentiale Komponente der Geschwindigkeit $\overline{\mathrm{V}}_{\mathrm{t}}$ und $\mathrm{B}=5.2$ eine Modellkonstante:

$\mathrm{y}^{+}=\frac{\rho \mathrm{u}_{\tau} \partial}{\mu} \mathrm{u}^{+}=\frac{\overline{\mathrm{v}}_{\mathrm{t}}}{\mathrm{u}_{\tau}} \mathrm{u}_{\tau}=\sqrt{\frac{\tau_{\mathrm{w}}}{\rho}}$

Gleichung 2

wobei $\mathrm{u}_{\tau}$ die Wandschubspannungsgeschwindigkeit der Wandschubspannung $\tau_{\mathrm{W}}$ ist. Unter Annahme nahezu paralleler Wandströmung kann die wandparallele Geschwindigkeitskomponente up und die Wandgeschwindigkeit $\mathrm{u}_{\mathrm{w}}$ mit der Wandschubspannungsgeschwindigkeit $\mathrm{u}_{\tau}$ in Beziehung gesetzt werden.

$\mathrm{up}_{\mathrm{p}}-\mathrm{u}_{\mathrm{w}}=\frac{\mathrm{u}_{\tau}}{\mathrm{K}} \ln \left(\mathrm{E}^{+}\right)$

Gleichung 3

Die Wandrauigkeit wird für glatte Wände mit $E=9.0$ angenommen, die Karman'sche Konstante ist $K=0.42$. Mit der Annahme, dass in der wandnächsten Zellschicht $\left(\right.$ Index $\left._{\mathrm{p}}\right)$ Produktion und Dissipation der turbulenten kinetischen Energie $k_{p}$ im Gleichgewicht sind, können $u_{\tau}$ und $\mathrm{y}^{+}$bestimmt werden.

$u_{\tau}=C_{\mu}^{1 / 4} \sqrt{k_{p},} y^{+}=\frac{\rho C_{\mu}^{1 / 4} \sqrt{k_{p}}}{\mu} \partial p$

Gleichung 4
Die Transportgleichung fuir die turbulente Dissipationsrate wird in der wandnächsten Zellschicht nicht gelöst, sondern wie folgt berechnet:

$\varepsilon_{p}=\frac{C_{\mu}^{1 / 4} k_{p}^{3 / 2}}{K \partial_{p}}$

Gleichung 5

Der dimensionslose Wandabstand $\mathrm{y}^{+}$ist vom Abstand $\partial \mathrm{p}$ des Zellmittelpunkts der wandnächsten Zelle abhängig. In der Literatur finden sich unterschiedliche Angaben über den Gültigkeitsbereich von $\mathrm{y}^{+}$; für die im Rahmen des Projektes eingesetzten Wandgesetze in der benutzten Simulationssoftware FLUENT wird ein Bereich von $30<\mathrm{y}^{+}<60$ angegeben.

\subsection{Standard Wandfunktion/Non-Equilibrium Wandfunktion}

FLUENT bietet zwei Wandfunktionen an, um die Lösungsvariablen von der Kernströmung zur Wand zu überbrïcken. Diese setzen sich aus einer Sammlung von halbempirischen Formeln und Funktionen zusammen. Die Wandfunktionen beinhalten:

- Wandgesetz für Durchschnittsgeschwindigkeit, Temperatur und andere skalare Größen,

- Formeln zur Berechnung der Turbulenz in Wandnähe.

Mit den „Standard Wall Functions“ und den „Non-Equilibrium Wall Functions“ stehen zwei Optionen zur Verfuigung. Beide Funktionen basieren auf einem Vorschlag von Launder und Spaldings logarithmischem Gesetz und haben sich in vielen Simulationsrechnungen bewährt. Die „Non-Equilibrium Wall Functions“ sind sensibilisiert, um Änderungen von Druckgradienten der Durchschnittsgeschwindigkeit besser auflösen zu können. Der Theorie nach wirkt sich dies insbesondere bei der Berechnung von Strömungen mit Ablösungen, Wiederanlegen und Aufprall positiv aus, da hier die Änderung der Druckgradienten sehr groß ist.

\subsection{Lösungsstrategie und Gitteradaption}

Die strömungstechnische Berechnung erfolgt auf iterativer Basis. Abbildung 2 zeigt einen typischen Verlauf der Residuen.

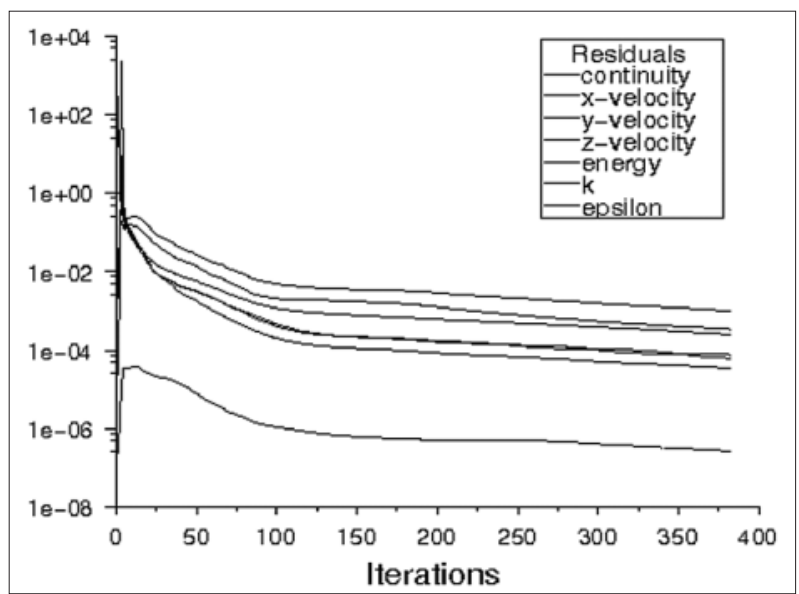

Abb. 2: Typischer Verlauf der Residuen 
Die Problematik bei der Berechnung ist, dass der Gültigkeitsbereich der Wandfunktion vom dimensionslosen Wandabstand $\mathrm{y}^{+}$abhängig ist. Dieser hängt wiederum von verschiedenen während der Simulationsrechnung zu ermittelnden Strömungsgrößen ab (vgl. Gleichung 2), die sich insbesondere mit der Reynoldszahl verändern. Deshalb ist es erforderlich, ausgehend von einer Initialisierungsrechnung, die wandnahe Gitterauflösung im Rahmen der iterativen Berechnung durch Adaptionsschritte in den Gültigkeitsbereich zu verschieben (Abb. 3).

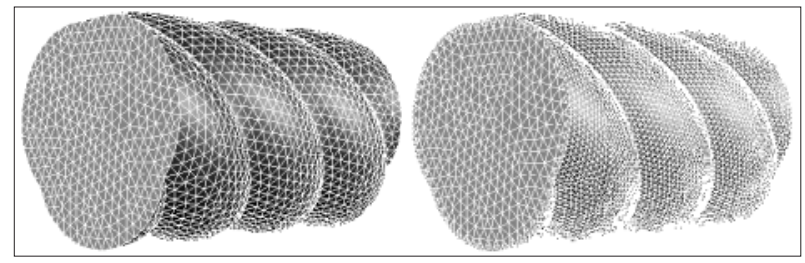

Abb. 3: Berechnungsgitter vor und nach Adaptierung der wandnahen Zellen

Sensivitätsrechnungen hinsichtlich der Abhängigkeit der Berechnungsergebnisse von der Auflösung des Gitters zeigen, dass die Ergebnisse innerhalb des Gültigkeitsbereichs von $\mathrm{y}+$ keineswegs identisch sind (Tab. 1).

\begin{tabular}{|l|l|l|}
\hline $\mathrm{y}+$ & $\mathrm{Nu}$ & $\Delta \mathrm{P}, \mathrm{Pa} / \mathrm{m}$ \\
\hline 31,7 & 198,0 & $5.198,6$ \\
\hline 39,2 & 178,5 & $4.802,3$ \\
\hline 46,4 & 160,1 & $4.677,6$ \\
\hline 50,9 & 150,3 & $4.179,5$ \\
\hline
\end{tabular}

Tab. 1: Berechnungsergebnisse für $N u$ und $\Delta P$ in Abhängigkeit von $y+$

Die Abweichungen können - wie im Beispiel gezeigt - leicht $25 \%$ betragen. Aus diesem Umstand ergibt sich neben der Erkenntnis, dass sich mit den zur Verfügung stehenden Mitteln nur Tendenzen bei der Ermittlung der Wärmeübertragungseigenschaften feststellen lassen, das Problem, eine verlässliche Vergleichbarkeit zwischen unterschiedlichen Berechnungsfällen herzustellen. Validierungen der Berechnungsergebnisse mit Messdaten haben verdeutlicht, dass die besten Übereinstimmungen zwischen Simulations- und Messwerten bei einem Wert $\mathrm{y}+$ von 30 liegen. Allerdings funktioniert die Adaption des Gitters nicht so selektiv, so dass dieser Wert niemals genau erreicht wird. Aus diesem Grund wurde eine Lösungsstrategie gewählt, bei der durch Adaptionsschritte möglichst mehrere Ergebnisse im Gültigkeitsbereich erhalten werden. Um eine Vergleichbarkeit der Simulati-

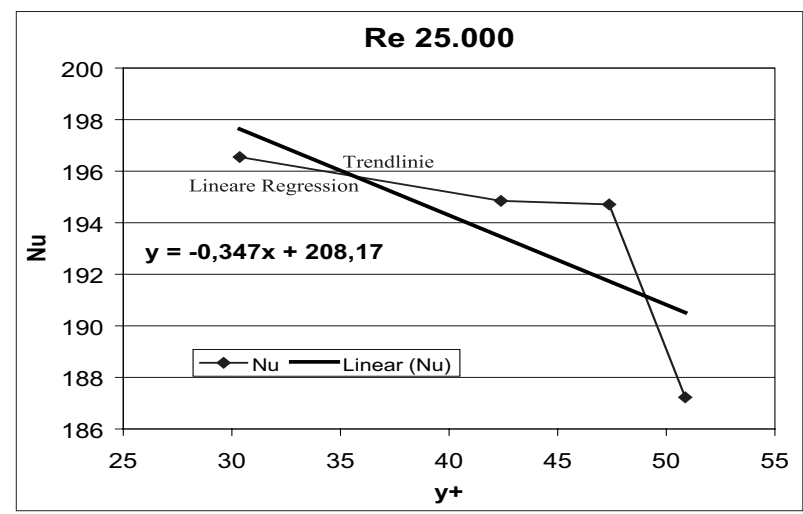

Abb. 4: Bestimmung der Nusselt-Zahl in Abhängigkeit von $y^{+}$ onsergebnisse zu ermöglichen, wird durch eine Lineare Regression anschließend der Wert für y+ von 30 aus den vorhandenen Ergebnissen extrapoliert. Nur durch dieses Lösungsverfahren wird es möglich, die Ergebnisse analytisch zu vergleichen (Abb. 4, 5).

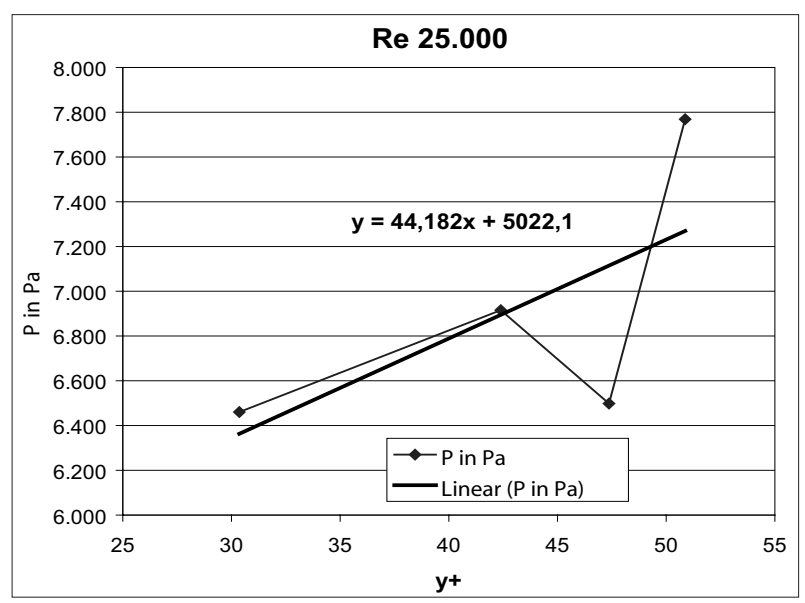

Abb. 5: Ermittlung des Druckverlustes in Abhängigkeit von $y^{+}$

Die spätere Auswertung der Ergebnisse verdeutlicht, dass durch die Anzahl der zur Verfuigung stehenden Werte im Gültigkeitsbereich und dem Abstand von y $+=30$ die Güte des extrapolierten Wertes schwankt.

\section{Simulationsrechnungen}

Da während der CFD-Untersuchungen noch keine Messergebnisse aus wärmetechnischen Untersuchungen für die IP-Tubes vorlagen, wurden vergleichende Berechnungen zwischen Glattrohren, Spiralrohren und IP-Tubes durchgefuihrt. Im Vordergrund standen dabei zwei wesentliche Aspekte. Zum einen soll neben der Validierung der Berechnungen fuir Glattrohre auch eine Validierung der Simulationsrechnungen für Rohre mit strukturierten Oberflächen erfolgen. Für Spiralrohre liegen eine Reihe von Messergebnissen vor, die als Datenmaterial für die Validierung verwendet werden können (Abb. 6). Nach der Validierung ist ein Vergleich der Spiralrohre mit IPTubes möglich. Zum anderen ist der Ausgangspunkt für die Entwicklung der IP-Tubes, dass sich ein günstigeres Verhältnis aus der Wärmeübertragungserhöhung zur Druckverlusterhöhung ergibt. Ein Vergleich mit Drallrohren soll zeigen, ob dieser energetische Vergleich tatsächlich vorteilhaft zugunsten der IP-Tubes ausfällt.

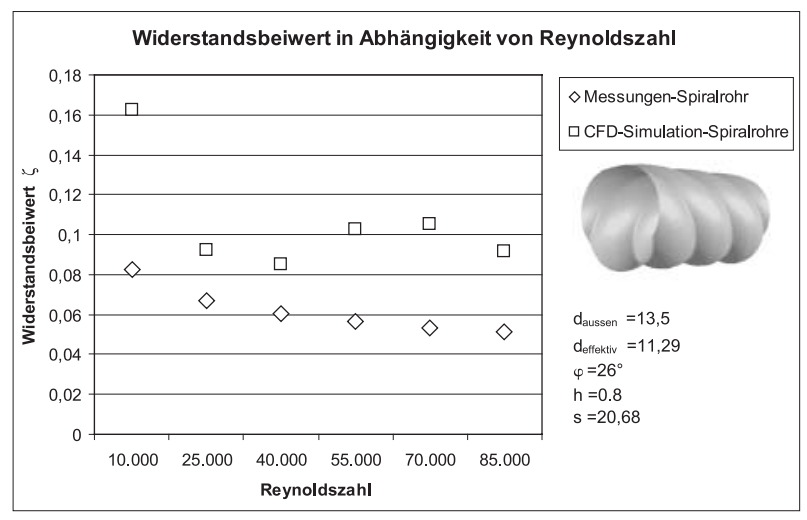

Abb. 6: Validierung der CFD-Simulation für Spiralrohr (Widerstandsbeiwert $\varphi$ ) 


\subsection{Vergleich von Glatt-/Spiralrohren und IP-Tubes für Abgasanlagen typische Randbedingungen}

Eine Schwierigkeit beim Vergleich von Glattrohren mit oberflächenstrukturierten Rohren liegt in der Definition des hydraulischen Durchmessers, der als Vergleichsdurchmesser herangezogen wird. FLUENT bietet die Möglichkeit, das Volumen des Untersuchungsgebietes zu berechnen. Aus dem Volumen und der Abschnittslänge wurde sowohl fuir das Spiralrohr wie auch für die IP-Tubes der mittlere Durchmesser als hydraulischer Durchmesser bestimmt und als Vergleichsdurchmesser festgelegt. Der Vergleich der Nusselt-Zahlen (Abb. 7) zeigt, dass die Erhöhung der Wärmeuibertragung des IP-Tubes deutlich über der des Spiralrohres liegt. Der Druckverlust nimmt beim IP-Tube allerdings nicht so stark zu wie beim Drallrohr (Abb. 8). Der energetische Vergleich (Abb. 9), welcher

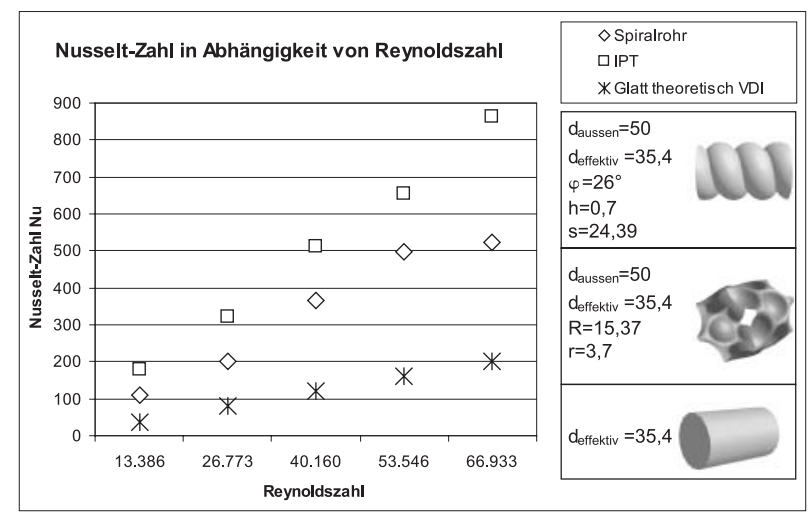

Abb. 7: Vergleich Nusselt-Zahlen für unterschiedliche Rohrtypen (abgasanlagentypische Randbedingungen)

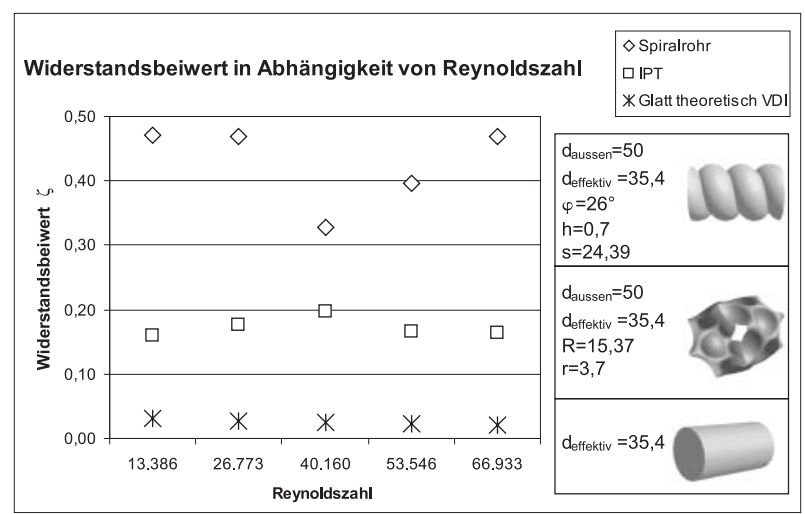

Abb. 8: Vergleich Widerstandsbeiwerte für unterschiedliche Rohrtypen (abgasanlagentypische Randbedingungen)

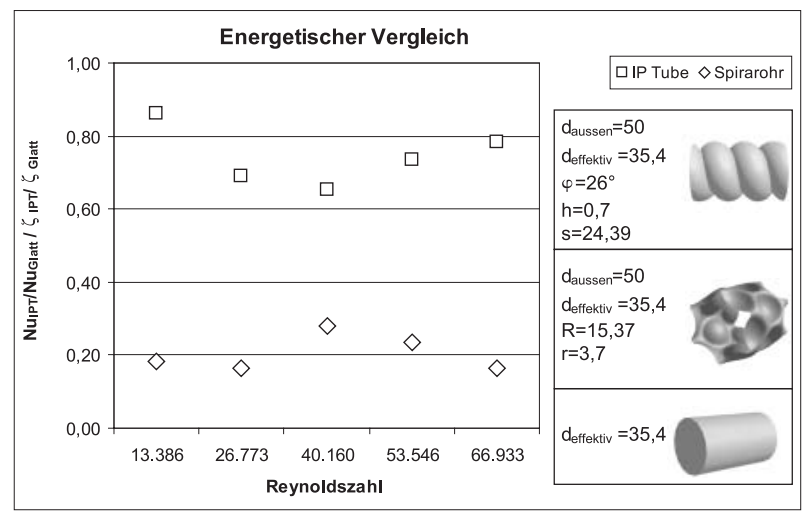

Abb. 9: Energetischer Vergleich für unterschiedliche Rohrtypen (abgasanlagentypische Randbedingungen)
Wärmeübertragung und Druckverlust (dargestellt in Form des dimensionslosen Widerstandbeiwertes) der oberflächenstrukturierten Rohre ins Verhältnis zum Glattrohr setzt, zeigt die deutliche Überlegenheit des IP-Tubes gegenüber Glatt- und Spiralrohr. Ein Wert von 1 für den energetischen Vergleich stellt das Optimum dar, d. h. die Erhöhung der Wärmeübertragung ist proportional zur Erhöhung des Druckverlustes.

\subsection{Formoptimierung}

Ziel der Formoptimierung ist, durch vergleichende Berechnungen die Einflüsse einzelner Strukturierungsdetails auf die Wärmeuibertragung zu ermitteln. Für die Formoptimierung wurden drei Parameter untersucht. Von diesen Parametern leitet sich die Grundform der Oberflächengeometrie der IP-Tubes ab. Die Parameter können direkt durch den Herstellungsprozess beeinflusst werden. Die Formoptimierung bewegt sich im Spannungsfeld von verbesserten Produkteigenschaften des umgeformten Halbzeugs (IP-Tube) und der Herstellbarkeit desselben. Im Fokus der verbesserten Produkteigenschaften ist die Erhöhung der Wärmeübertragung das wesentliche Ziel. Ziel ist natürlich auch, eine möglichst einfach herstellbare Form zu finden. Kann der Aufwand der Strukturierung, z. B. durch eine geringere Anzahl an Nebenformen, minimiert werden, sinken die Umformkosten, woraus eine deutlich verbesserte Wettbewerbsfähigkeit im Vergleich zu alternativen Rohrhalbzeugen mit strukturierten Oberflächen resultiert. Variiert wurden die Tiefe, axialer und rotatorischer Versatz der Nebenformen. Das Versuchsprogramm ist in Tabelle 2 (siehe Seite 5) zusammengefasst.

\subsection{Tiefe der Nebenformen}

Es wurden drei verschiedene Nebenformtiefen untersucht (1 mm, $3 \mathrm{~mm}, 5 \mathrm{~mm}$ ). Die Berechnungen zum Einfluss der Nebenformtiefe zeigen, dass Wärmeübertragung und Druckverlust mit der Tiefe der Nebenform zunehmen.

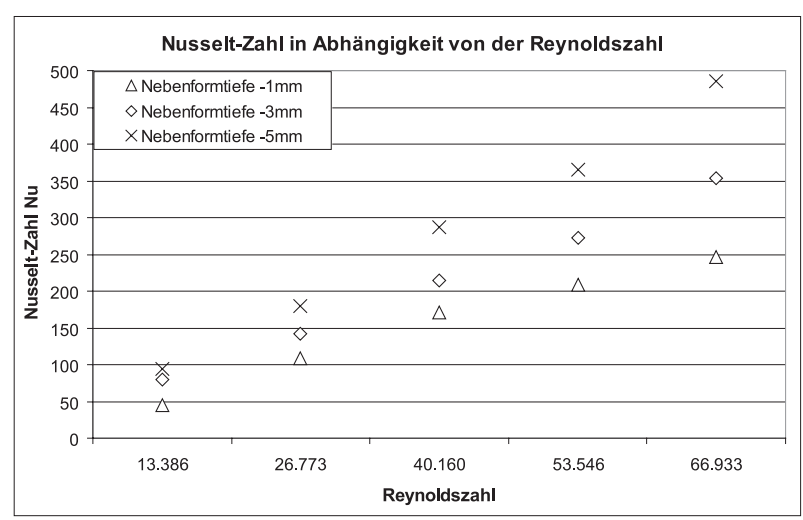

Abb. 10: Vergleich der Nusselt-Zahlen für unterschiedliche Nebenformtiefen

Betrachtet man den Einfluss der Beultiefe auf Wärmeuibertragung und Druckverlust, ergibt sich bei einer arithmetischen Mittelung über die errechneten Werte bei unterschiedlichen Reynoldszahlen folgendes generelles Bild. Für den untersuchten Wertebereich erhöht sich die Nusselt-Zahl pro Millimeter zusätzlicher Tiefe der Nebenformen um den Faktor 1,18; der Druckverlust erhöht sich um das 1,41 fache. 


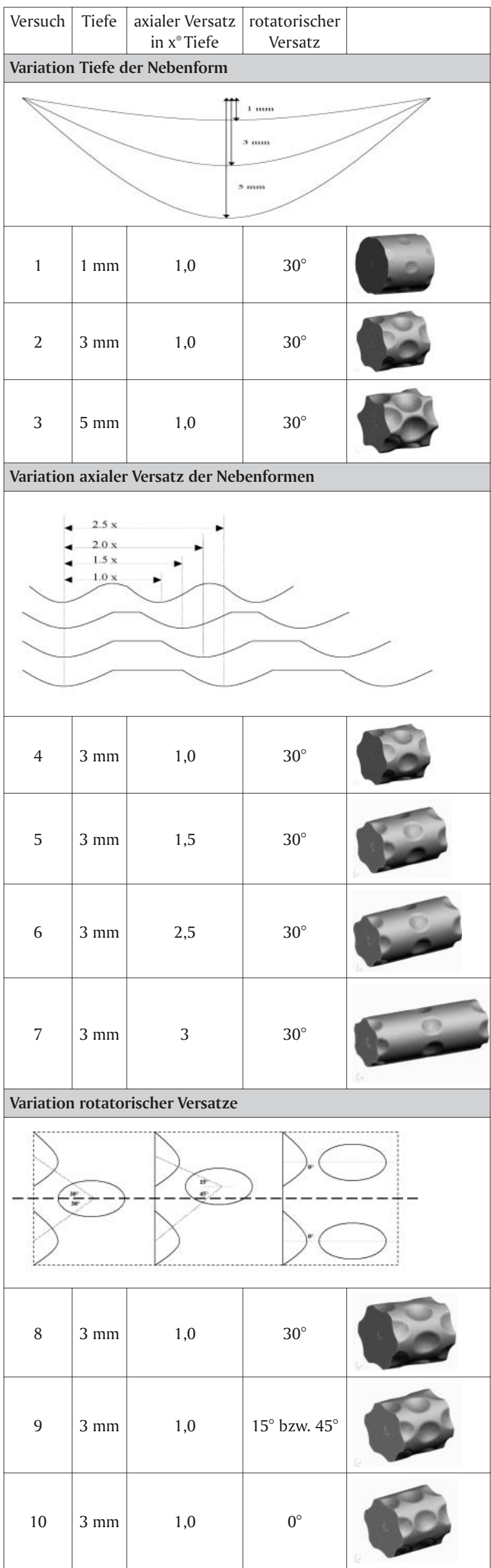

Tab. 2: Versuchsprogramm zur Formoptimierung der IP-Tubes

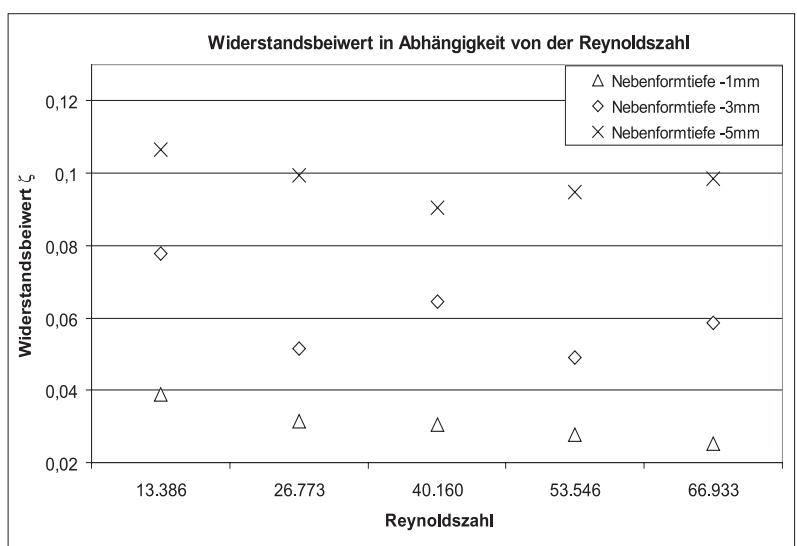

Abb. 11: Vergleich der Widerstandsbeiwerte für unterschiedliche Nebenformtiefen

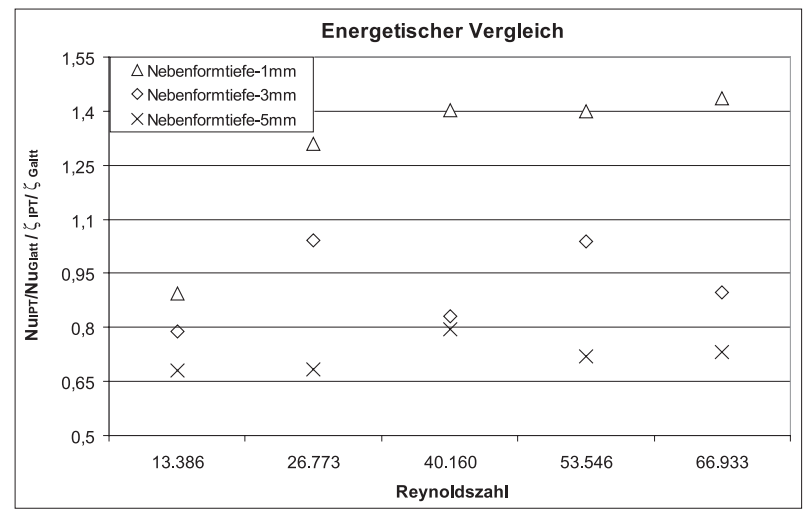

Abb. 12: Energetischer Vergleich für unterschiedliche Nebenformtiefen

\subsection{Axialer Versatz der Nebenformen}

Es wurden vier Varianten des axialen Versatzes untersucht. Ausgangspunkt für die Variationsrechnungen ist die Länge der Nebenform. Bei der Basisvariante aller Untersuchungen entspricht der Abstand zwischen den Mittelpunkten der Nebenform der Länge der Nebenform (axialer Versatz $=1)$. Dieser Versatz wurde in den Stufen 1,5, 2,0, 2,5 variiert. Eine Veränderung des axialen Versatzes hat im untersuchten Wertebereich keinen signifikanten Einfluss auf Wärmeübertragung und Druckverlust. Für den untersuchten Wertebereich erhöht sich die Nusselt-Zahl pro 0,5fach erhöhten Abstand zwischen den Nebenformen nicht; der Druckverlust verringert sich um das 0,97 fache.

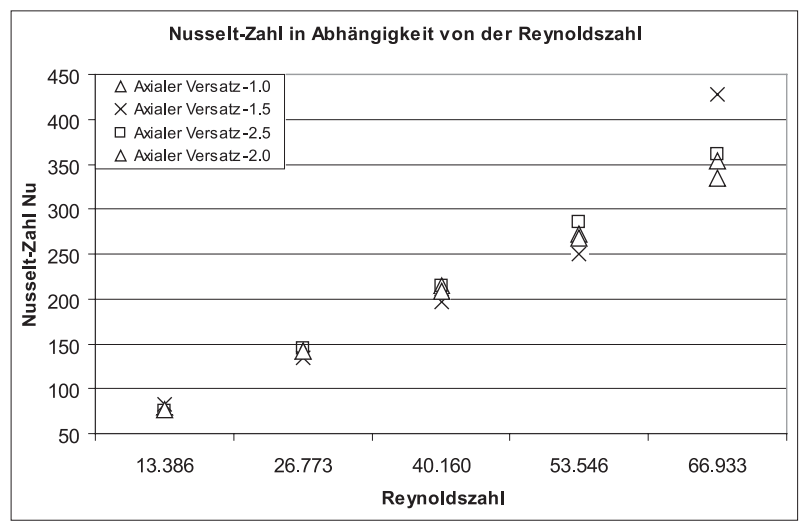

Abb. 13: Vergleich der Nusselt-Zahlen für unterschiedlichen axialen Versatz der Nebenformen 


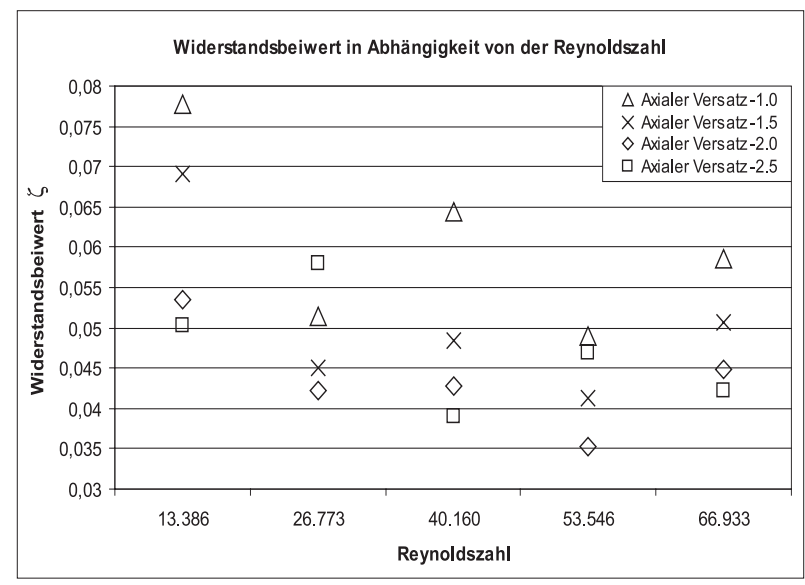

Abb. 14: Vergleich der Widerstandsbeiwerte für unterschiedlichen axialen Versatz der Nebenformen

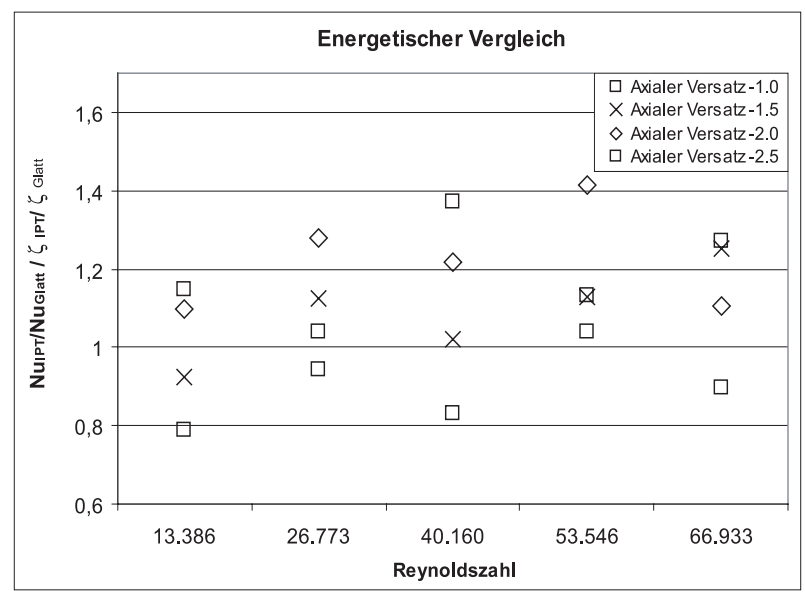

Abb. 15: Energetischer Vergleich für unterschiedlichen axialen Versatz der Nebenformen

\subsection{Rotatorischer Versatz der Nebenformen}

Der rotatorische Versatz der Nebenformen wurde in drei Stufen mit einer Variation des Versatzes um jeweils $15^{\circ}$ untersucht. Der Versatz hat keinen signifikanten Einfluss auf Wärmeübertragung und Druckverlust für den untersuchten Wertebereich. Es ergeben sich nur vernachlässigbar geringe Änderungen für Wärmeübertragung und Druckverlust.

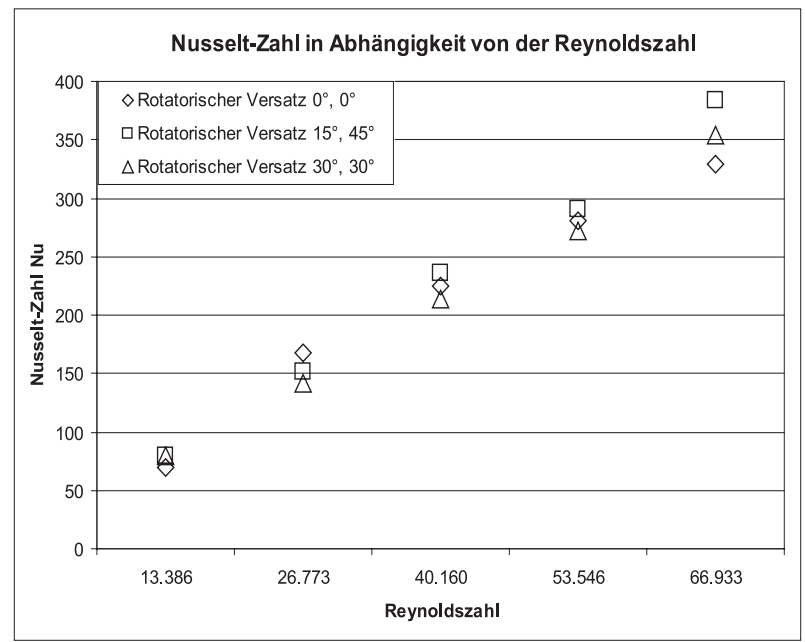

Abb. 16: Vergleich der Nusselt-Zahlen für unterschiedlichen rotatorischen Versatz der Nebenformen

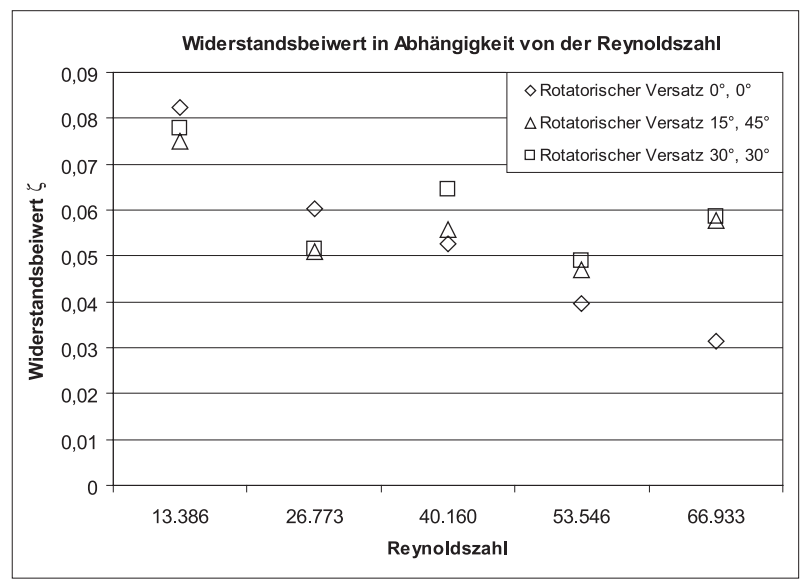

Abb. 17: Vergleich der Widerstandsbeiwerte für unterschiedlichen rotatorischen Versatz der Nebenformen

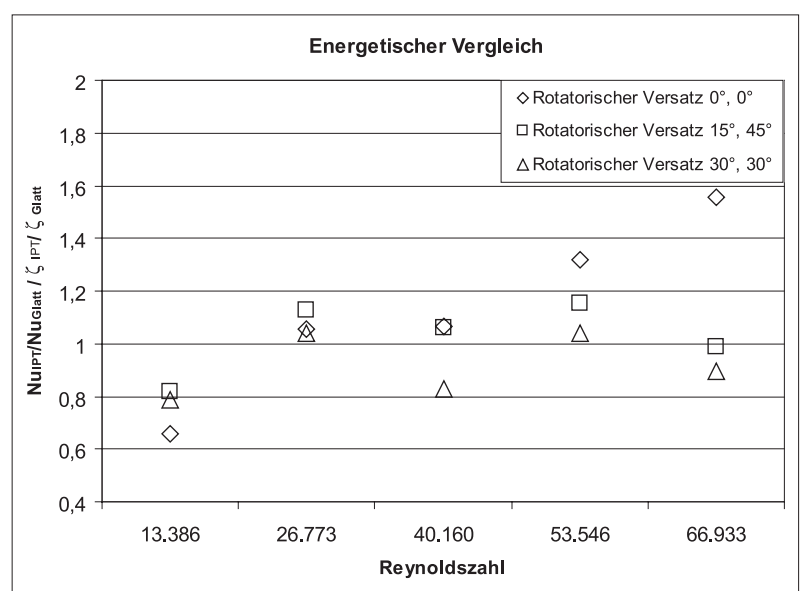

Abb. 18: Energetischer Vergleich für unterschiedlichen rotatorischen Versatz der Nebenformen

\subsection{Beschreibung strömungstechnischer Effekte auf Basis der Simulationsergebnisse}

Erwartungsgemäß ist die Tiefe der Nebenform der entscheidende Parameter zur Beeinflussung des Wärmeübertragungskoeffizienten. Durch eine tiefere Ausprägung der Nebenform nimmt die Störung der Strömung zu, woraus ein verstärkter Austausch zwischen dem Kern der Strömung und der wandnahen Grenzschicht erfolgt (Abb. 19). Es erfolgt eine periodische Kontraktion und Dillatation der Strömung.

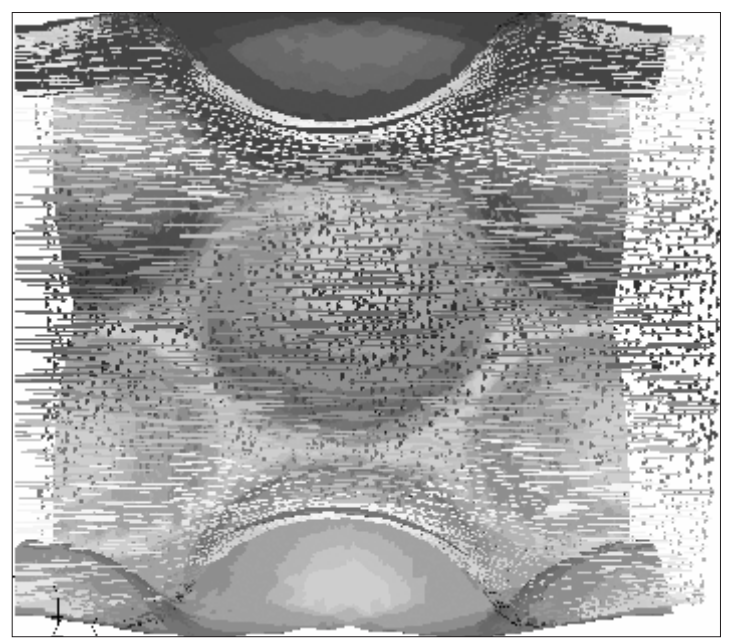

Abb. 19: Vektoren der Geschwindigkeit 
Betrachtet man die Verteilung des Wärmeübertragungskoeffizienten auf der Rohroberfläche (Abb. 20) für den Basisfall (Nebenformtiefe $=3 \mathrm{~mm}$; axialer Versatz $=$ 1,0 , rotatorischer Versatz $=30^{\circ}$ ), wird deutlich, dass die höchste Wärmeübertragung im Staupunkt der Strömung an der Nebenform erfolgt. Im Abströmbereich der Nebenform nimmt diese deutlich ab. Der Übergangsbereich zur nächsten Nebenform leistet nahezu keinen Beitrag zur Gesamtwärmeübertragung. Dies liegt in der Wiederanleglänge der Strömung begründet: In diesem Bereich bildet sich eine Rezirkulationszone aus.

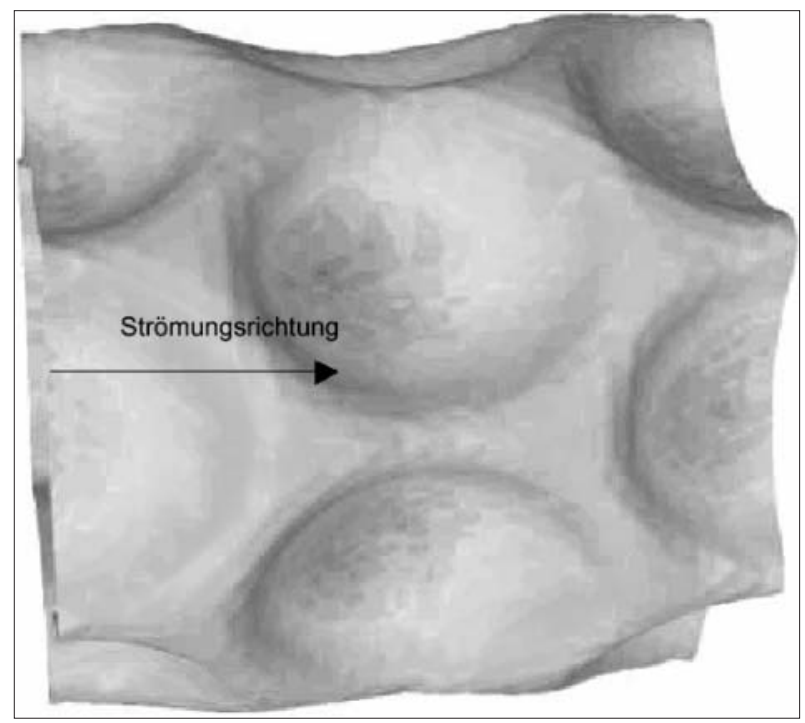

Abb. 20: Konturen des Wärmeübertragungskoeffizienten

Die Erhöhung des axialen Versatzes bis zum 2,5fachen der Nebenformlänge hat bei der untersuchten Nebenformtiefe von $3 \mathrm{~mm}$ kaum einen Einfluss auf die Wärmeübertragung. Der Druckverlust reduziert sich geringfuigig. Je geringer der axiale Versatz ist, desto ausgeprägter wird die Kernströmung und die Bereiche zwischen den Nebenformen werden kaum genutzt. Dadurch wird die Anströmfläche im Staupunkt geringer. Wird der axiale Versatz erhöht, führt dies zu zwei die Wärmeübertragung beeinflussenden Effekten: Die Anzahl der Nebenformen pro Längeneinheit nimmt ab, wodurch die Staupunkte mit erhöhter Wärmeübertragung abnehmen und sich die Wärmeübertragung reduziert. Mit zunehmendem Versatz legt sich die Strömung wieder an den äußeren Rohrmantel an. Dadurch erhöht sich die Staupunktfläche an der in Strömungsrichtung folgenden Nebenform, wodurch der Bereich erhöhter Wärmeübertragung an der jeweiligen Nebenform zunimmt. Zudem wird die wandnahe Strömung stärker in den Strömungskern abgelenkt, wodurch die Vermischung mit dem Strömungskern intensiviert wird. Die Verminderung der Wärmeübertragung durch die geringere Anzahl an Nebenformen kann somit durch eine Erhöhung der Wärmeübertragung an den Nebenformen und eine intensivierte Vermischung mit dem Strömungskern bei $3 \mathrm{~mm}$ Nebenformtiefe kompensiert werden. Dieser Effekt ist selbstverständlich eine Funktion der Nebenformtiefe. D. h. mit zunehmender Nebenformtiefe kann der axiale Abstand zwischen den Nebenformen erhöht werden, ohne dass es zu einer signifikanten Verringerung der Wärmeübertragung kommt.

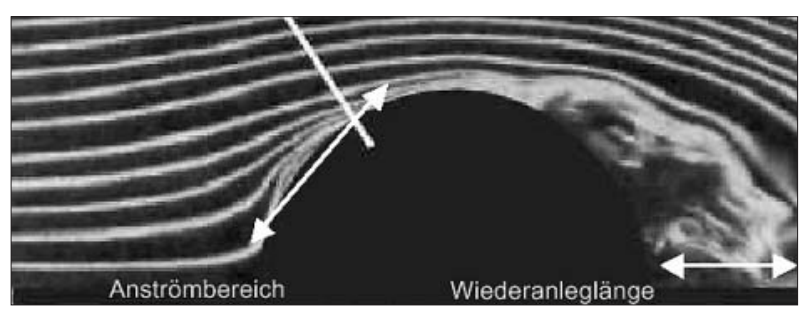

Abb. 21: Staupunkt der Anströmung und Wiederanlegen der Strömung für eine zylindrische Fläche

Eine Änderung des rotatorischen Versatzes hat bei dem Basisfall keinen signifikanten Einfluss auf die Wärmeübertragung. Die Quervermischung scheint keinen dominanten Einfluss auf die Wärmeübertragung zu haben, da die Strömung sich auf den Kern des Rohres verdichtet. Anzunehmen ist, dass der Einfluss des rotatorischen Versatzes auf die Wärmeübertragung mit zunehmendem axialen Versatz und verringerter Nebenformtiefe zunehmen wird.

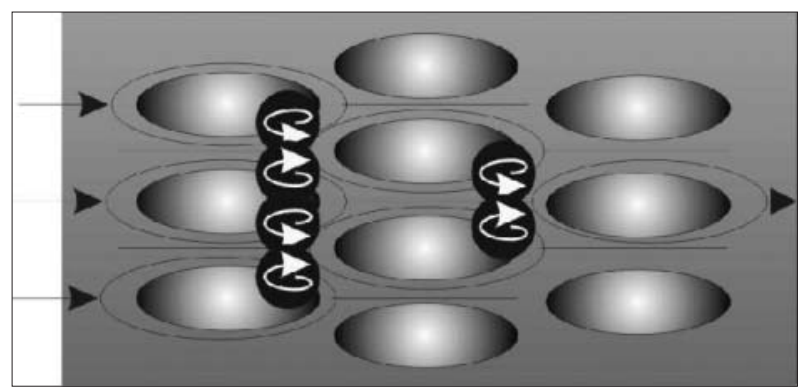

Abb. 22: Quervermischung durch die Nebenformen

\section{Zusammenfassung}

Ziel der Untersuchungen war es, durch Computational Fluid Dynamics (CFD)-Simulationen das Strömungs- und Wärmeübertragungsverhalten von oberflächenstrukturierten Rohrabschnitten (Industrial Power Tubes, kurz IP-Tubes) fuir den Einsatz als Abgaswärmeüberträger in Kfz-Abgasanlagen zu testen. Die Simulationen haben verdeutlicht, dass mit vertretbarem Aufwand bezüglich der Rechenleistung keine quantitativ verwertbaren Ergebnisse erzielt werden können. Turbulenzmodelle, Wandfunktionen und die Gitterauflösung im wandnahen Bereich haben einen signifikanten Einfluss auf das Ergebnis. Berechnungen im Gültigkeitsbereich eines Wandgesetzes für den dimensionslosen Wandabstand $y+(G u ̈ i l t i g k e i t s b e r e i c h ~ 30-60)$ können in Abhängigkeit des durch Gitteradaption eingestellten Wertes von y+ leicht Abweichungen von 25 \% haben. Allerdings zeigen die Rechenergebnisse einen klaren Trend. Je näher das adaptierte Gitter an dem Wert y+ 30 liegt, desto höher ist die berechnete Nusselt-Zahl und desto näher liegt der Wert an den realen Werten. Aus diesem Grund wurde eine Lösungsstrategie gewählt, bei der durch Adaptionsschritte möglichst mehrere Ergebnisse im Gültigkeitsbereich erhalten werden. In der Regel konnten durch Gitteradaptionen und anschließende Rechenläufe 5 - 6 Ergebnisse im Gültigkeitsbereich errechnet werden. Um eine Vergleichbarkeit der Simulationsergebnisse zu ermöglichen, wurde durch eine Lineare Regression anschließend der Wert für y+ von 30 aus den vorhandenen Ergebnissen extrapoliert. Tests mit unterschiedlichen 
Kombinationen aus Wandfunktion und Turbulenzmodellen haben gezeigt, dass bei vertretbarem Aufwand das klassische k-e-Modell in Verbindung mit den Standard-Wandfunktionen verwertbare Trends liefert. Um quantitativ verwertbare Ergebnisse zu erhalten, wird die viskose Unterschicht durch ein hinreichend feines Gitter aufgelöst (Near Wall Model Approach). Hierzu ist es notwendig, die viskose Unterschicht zumindest mit einer Schichtdicke von ca. zehn Zellen aufzulösen, da die Gradienten in Wandnähe sehr stark sind und nur durch ein extrem feines Gitter aufgelöst werden können. Mit zunehmender Reynoldszahl nimmt die Schichtdicke der viskosen Unterschicht durch Verdrängung ab, wodurch das wandnahe Gitter immer feiner werden muss, was mit einem starken Anstieg des Berechnungsaufwandes einhergeht.

Im Vordergrund der Untersuchung standen der qualitative Vergleich der IP-Tubes mit Wettbewerbsprodukten (Drallrohr) und die Herausarbeitung der Einfluisse unterschiedlicher Strukturierungsdetails der IP-Tubes auf die Wärmeübertragung. Der Vergleich zwischen IP-Tube und Drallrohr zeigt, dass die Erhöhung der Wärmeübertragung des IP-Tubes gegenuiber einem glatten Vergleichsrohr deutlich über der des Spiralrohres liegt. Der Druckverlust nimmt beim IP-Tube allerdings nicht so stark zu wie beim Drallrohr. Der energetische Vergleich, welcher Wärmeübertragung und Druckverlust (dargestellt in Form des dimensionslosen Widerstandbeiwertes) der oberflächenstrukturierten Rohre ins Verhältnis zum Glattrohr setzt, zeigt die deutliche Überlegenheit des IP-Tube (Mittelwert $=0,74$ ) gegenuiber dem Spiralrohr (Mittelwert $=0,2$ ). Der Wert 1 für den energetischen Vergleich kennzeichnet eine proportionale Zunahme von Wärmeübergang und Druckverlust, was den optimalen Fall darstellt. Für die Formoptimierung wurden drei Parameter untersucht. Von diesen Parametern leitet sich die Grundform der Oberflächengeometrie der IP-Tubes ab. Variiert wurden die Tiefe, axialer und rotatorischer Versatz der Nebenformen.

Es wurden drei verschiedene Nebenformtiefen untersucht (1 mm, $3 \mathrm{~mm}, 5 \mathrm{~mm}$ ). Die Berechnungen zum Einfluss der Nebenformtiefe zeigen, dass Wärmeübertragung und Druckverlust mit der Tiefe der Nebenform zunehmen. Für den untersuchten Wertebereich erhöht sich die Nusselt-Zahl pro Millimeter zusätzlicher Tiefe der Nebenformen um den Faktor 1,18; der Druckverlust erhöht sich um das 1,41fache. Es wurden vier Varianten des axialen Versatzes untersucht. Ausgangspunkt für die Variationsrechnungen ist die Länge der Nebenform. Bei der Basisvariante aller Untersuchungen entspricht der Abstand zwischen den Mittelpunkten der Nebenform der Länge der Nebenform (axialer Versatz $=1$ ). Dieser Versatz wurde in den Stufen 1,5, 2,0, 2,5 variiert. Eine Veränderung des axialen Versatzes hat im untersuchten Wertebereich keinen signifikanten Einfluss auf Wärmeübertragung und Druckverlust. Für den untersuchten Wertebereich erhöht sich die Nusselt-Zahl pro 0,5fach erhöhten Abstand zwischen den Nebenformen nicht; der Druckverlust verringert sich um das 0,97fache. Der rotatorische Versatz der Nebenformen wurde in drei Stufen mit einer Variation des Versatzes um jeweils $15^{\circ}$ untersucht. Der Versatz hat keinen signifikanten Einfluss auf Wärmeübertragung und Druckverlust für den untersuchten Wertebereich. Es ergeben sich nur vernachlässigbar geringe Änderungen für Wärmeübertragung und Druckverlust.

\section{Fazit}

Die Formoptimierung bewegt sich im Spannungsfeld von verbesserten Produkteigenschaften des umgeformten Halbzeugs (IP-Tube) und der Herstellbarkeit desselben. Im Fokus der verbesserten Produkteigenschaften ist die Erhöhung der Wärmeübertragung das wesentliche Ziel. Ziel ist natuirlich auch, eine möglichst einfach herstellbare Form zu finden. Kann der Aufwand der Strukturierung, z. B. durch eine geringere Anzahl an Nebenformen, minimiert werden, sinken die Umformkosten, woraus eine deutlich verbesserte Wettbewerbsfähigkeit im Vergleich zu alternativen Rohrhalbzeugen mit strukturierten Oberflächen resultiert. Die Erkenntnis, dass sich vergleichbare Wärmeübertragungskoeffizienten bei einer Erhöhung des axialen Versatzes ergeben, hat unmittelbaren Einfluss auf die Fertigungskosten der IP-Tubes. Eine optimierte Form wird durch ausgeprägte Nebenformen (Tiefe der Nebenform) und einen hohen axialen Versatz erreicht. Da bisher die Nebenformen abschnittsweise als Kranz in die Rohre gedrüickt werden und längere Abschnitte des Rohres unstrukturiert verbleiben können, reduziert sich der Aufwand nahezu linear mit der Länge des unstrukturierten Bereiches. Die Umformkosten können also bei Beruicksichtigung der Untersuchungsergebnisse signifikant reduziert werden. Zur Verifizierung der CFDSimulationen ist es sicherlich erforderlich, wärmetechnische Untersuchungen für die avisierten Applikationen durchzufuihren, um die Wärmeübertragungskoeffizienten genau bestimmen zu können.

\section{Autoren}

\section{Prof. Dr.-Ing. Udo Hellwig}

Technische Fachhochschule Wildau

Fachbereich Ingenieurwesen/Wirtschaftsingenieurwesen

Tel. +493375 508-170

Fax +493375 508-127

E-Mail: hellwigu@vt.tfh-wildau.de

Dipl.-Ing. (FH) Hartwig Nikolaus

ERK Eckrohrkessel GmbH

Großbeerenstraße 71, 10963 Berlin

Tel. $+4930897746-0$

Fax +4930897746-46

E-Mail: hnikolaus@eckrohrkessel.com 